\title{
PHASE PORTRAITS OF THE QUADRATIC POLYNOMIAL LIÉNARD DIFFERENTIAL SYSTEMS
}

\author{
MÁRCIO R. A. GOUVEIA ${ }^{1}$, JAUME LLIBRE² AND LUCI ANY ROBERTO ${ }^{1}$
}

\begin{abstract}
We classify the global phase portraits in the Poincaré disc of the quadratic polynomial Liénard differential systems

$$
\dot{x}=y, \quad \dot{y}=(a x+b) y+c x^{2}+d x+e,
$$

where $(x, y) \in \mathbb{R}^{2}$ are the variables and $a, b, c, d, e$ are real parameters.
\end{abstract}

\section{INTRODUCTION}

A quadratic polynomial differential system is a system of the form

$$
\dot{x}=P(x, y), \quad \dot{y}=Q(x, y),
$$

where $P$ and $Q$ are polynomials in the variables $x$ and $y$, and the maximum of the degrees of $P$ and $Q$ is two.

The quadratic polynomial differential systems and their applications have been studied intensively these last thirty years, see for instance the exhaustive bibliography about these systems in the books of Reyn [37] and Ye Yanqian [44]. More concretely, classes of quadratic systems that have been studied are: homogeneous (see [13, 31, 33]), bounded (see $[15,11,17]$ ), having a star nodal point (see [6]), chordal (see [20, $21]$ ), with a weak focus of second or third order (see $[4,5,29,26]$ ), with four infinite critical points and one invariant straight line (see [39]), Hamiltonian (see [2]), gradient (see [10]), having a focus and one antisaddle (see [3]), integrable quadratic systems using Carleman and Painlevé tools (see [22]), having a center (see [41] and [29]), ...

On the other hand a polynomial Liénard differential system is a system of the form

$$
\dot{x}=y, \quad \dot{y}=f(x) y+g(x),
$$

where $f$ and $g$ are polynomials in the variable $x$.

2000 Mathematics Subject Classification. 37C29, 37D45.

Key words and phrases. Quadratic system, Liénard system, Poincaré compactification, global phase portraits. 
The polynomial Liénard differential systems and their applications also have been analyzed by many authors these recent years. Thus some authors studied their limit cycles (see for instance [14, 16, 19, 23, $24,36,38]$ ), or their algebraic limit cycles (see $[25,28,34]$ ), or their invariant algebraic curves (see $[8,7,45]$ ), or their canard limit cycles (see [40]), or the shape of their limit cycles (see [42]), or the period function of their centers (see [43]), or their integrability (see [9, 27]).

Roughly speaking the Poincaré disc $\mathbb{D}^{2}$ is the closed unit disc centered at the origin of coordinates of $\mathbb{R}^{2}$, where its interior is identified with $\mathbb{R}^{2}$ and its boundary $\mathbb{S}^{1}$ is identified with the infinity of $\mathbb{R}^{2}$, i.e. in the plane we can go to infinity in as many as we directed points for the circle $\mathbb{S}^{1}$. So a polynomial differential system in $\mathbb{R}^{2}$ (i.e. in the interior of $\mathbb{D}^{2}$ ) is extended analytically to the whole $\mathbb{D}^{2}$. In this way we can study the dynamics of the differential system in a neighborhood of infinity. For details on the Poincaré disc see section 3, and chapter 5 of [18].

Up to now the phase portraits in the Poincaré disc of the quadratic polynomial Liénard differential systems have not been studied, their study is the goal of this paper. More precisely, our objective is to classify the different topological phase portraits in the Poincaré disc of the systems

$$
\dot{x}=y, \quad \dot{y}=(a x+b) y+c x^{2}+d x+e,
$$

where $(x, y) \in \mathbb{R}^{2}$ are the variables and $a, b, c, d, e$ are real parameters.

We denote by $\mathcal{X}=\left(y,(a x+b) y+c x^{2}+d x+e\right)$ the vector field defined by system (1). We observe that since we are interested in the quadratic polynomial Liénard differential systems we must assume that the parameters satisfy $a^{2}+c^{2} \neq 0$ and $a^{2}+b^{2} \neq 0$ in order to avoid the non-quadratic systems and in order to be of Liénard type respectively. Moreover we need that $c^{2}+d^{2}+e^{2} \neq 0$, otherwise $y=0$ is a straight line filled of equilibria and the system can be reduced to a linear one.

Two phase portraits in the Poincaré disc $\mathbb{D}^{2}$ are topologically equivalent if there exists a homeomorphism $h: \mathbb{D}^{2} \rightarrow \mathbb{D}^{2}$ which sends orbits of one of the phase portraits into orbits of the other phase portrait, preserving or reversing the orientation of all the orbits.

Our main result is the following one.

Theorem 1. A quadratic polynomial Liénard differential system (1) has a phase portrait in the Poincaré disc topologically equivalent to one 
of the phase portraits of Figures 3, 4, 5, 6, 7, 8, 9. That is, there are 17 different topological phase portraits in the Poincaré disc for system (1).

In order to prove Theorem 1 we will make use of the normal forms of system (1) in section 2, which simplify in somehow the envolved calculations. Afterthat in section 3 we analyze the appearance and behaviour of the infinite singular points at those normal forms. Finally in section 4 we study the appearance and behaviour of the finite singular points in each normal form given in section 2 , and the proof of Theorem 1 is given in section 4 .

For studying the local phase portraits at the finite and infinite singular points of the compactified quadratic polynomial Liénard differential systems we use notations and results presented in chapters 2, 3 and 5 of [18]. For classifying the global phase portraits of the quadratic polynomial Liénard differential systems in the plane $\mathbb{R}^{2}$ extended to infinity we follow the notations and results on Poincaré disc in chapter 5 in [18], and with the result due to Markus [30], Neumann [32] and Peixoto [35], which guarantees that we only need to classify only all the different configurations of separatrices of the compactified quadratic polynomial Liénard differential systems, in order to obtain their phase portraits in the Poincaré disc.

\section{NORMAL FORMS}

The next result will simplify the study of the phase portraits of system (1) in the Poincaré disc.

Proposition 2. All systems (1) are topologically equivalent to one of the normal forms $(i)-(v i)$ in Table 2 , where $A, B, C, D, E, G, H, I$ are paremeters.

Proof. Fixed $\alpha, \beta, \gamma \neq 0$, after the linear change of coordinate $(x, y) \mapsto$ $(\alpha X, \beta Y)$ and the time rescaling $t \mapsto \gamma T$, system (1) becomes

(2) $\frac{d X}{d T}=\frac{\gamma \beta}{\alpha} Y, \quad \frac{d Y}{d T}=c \frac{\gamma \alpha^{2}}{\beta} X^{2}+a \alpha \gamma X Y+d \frac{\alpha \gamma}{\beta} X+b \gamma Y+e \frac{\gamma}{\beta}$.

We study six cases separately. In each case, we assume

$$
\frac{\gamma \beta}{\alpha}=1 \text {. }
$$


TABLE 1. Normal forms for system (1).

\begin{tabular}{|c|l|l|}
\hline & System (1) & Normal Form \\
\hline (i) & $a \neq 0 c \neq 0$ & $\dot{x}=y, \dot{y}=x^{2}+x y+A x+B y+C$ \\
\hline (ii) & $a=0, b c \neq 0$ & $\dot{x}=y, \dot{y}=x^{2}+y+D x+E$ \\
\hline (iii) & $a b \neq 0, c=0$ & $\dot{x}=y, \dot{y}=x y+y+G x+H$ \\
\hline (iv) & $a \neq 0, b=c=0, d>0$ & $\dot{x}=y, \dot{y}=x y+x+I$ \\
\hline (v) & $a \neq 0, b=c=0, d<0$ & $\dot{x}=y, \dot{y}=x y-x+I$ \\
\hline (vi) & $a \neq 0, b=c=d=0, e \neq 0$ & $\dot{x}=y, \dot{y}=x y+1$ \\
\hline
\end{tabular}

Case (i): $a c \neq 0$. After the above change of coordinate we can take (3), $c \frac{\gamma \alpha^{2}}{\beta}=1$ and $a \alpha \gamma=1$. These conditions are satisfied if $\alpha=\frac{c}{a^{2}}$, $\beta=\frac{c^{2}}{a^{3}}$ and $\gamma=\frac{a}{c}$. System (2) becomes

$$
\frac{d X}{d T}=Y, \quad \frac{d Y}{d T}=X^{2}+X Y+A X+B Y+C,
$$

where $A=\frac{d}{c^{2}}, B=\frac{a b}{c}$ and $C=\frac{a^{4} e}{c^{3}}$.

Case (ii): $a=0$ and $b c \neq 0$. Then we assume (3), $c \frac{\gamma \alpha^{2}}{\beta}=1$ and $b \gamma=1$, and the solution is $\alpha=\frac{b^{2}}{c}, \beta=\frac{b^{3}}{c}$ and $\gamma=\frac{1}{b}$. System (2) becomes

$$
\frac{d X}{d T}=Y, \quad \frac{d Y}{d T}=X^{2}+D X+Y+E,
$$

where $D=\frac{d c}{b^{5}}$ and $E=\frac{e c}{b^{4}}$.

Case (iii): $a b \neq 0$ and $c=0$. Then we assume (3), $a \alpha \gamma=1$ and $b \gamma=1$. System (2) becomes

$$
\frac{d X}{d T}=Y, \quad \frac{d Y}{d T}=X Y+Y+G X+H
$$


where $G=d / b^{2}$ and $H=e b / a$.

Case (iv): $a \neq 0, b=c=0$ and $d>0$. Then we assume (3), $a \alpha \gamma=1$ and $d \frac{\alpha \gamma}{\beta}=1$. System (2) becomes

$$
\frac{d X}{d T}=Y, \quad \frac{d Y}{d T}=X Y+X+I,
$$

where $I= \pm \frac{a e}{d^{3 / 2}}$.

Case (v): $a \neq 0, b=c=0$ and $d<0$. Then we assume (3), $a \alpha \gamma=1$ and $d \frac{\alpha \gamma}{\beta}=-1$. System (2) becomes

$$
\frac{d X}{d T}=Y, \quad \frac{d Y}{d T}=X Y-X+I
$$

where $I= \pm \frac{a e}{|d|^{3 / 2}}$.

Case (vi): $a \neq 0, b=c=d=0$ and $e \neq 0$. Then we assume (3), $a \alpha \gamma=1$ and $e \frac{\gamma}{\beta}=1$. System (2) becomes

$$
\frac{d X}{d T}=Y, \quad \frac{d Y}{d T}=X Y+1
$$

This complete the proof of the proposition.

\section{INFINITE SINGULAR POINTS}

In this section we study the infinite singular points of the quadratic polynomial Liénard differential systems using the notation and results of chapter 5 in [18].

3.1. Infinite singular points in the local charts $U_{1}$ and $V_{1}$. From equation (5.2) in [18], we obtain that the expression of the Poincaré compactification $p(\mathcal{X})$ of system $(1)$ in the local chart $U_{1}$ is

$$
\begin{aligned}
& \dot{u}=c+a u+d v+b u v+e v^{2}-u^{2} v, \\
& \dot{v}=-u v^{2} .
\end{aligned}
$$

Proposition 3. If $a=0$ there is no infinite singular points in $U_{1}$. If $a \neq 0$ there is a unique equilibrium point at $U_{1}$ and another unique equilibrium point at $V_{1}$ as described in Figure 1. 
Proof. Taking $v=0$ in (4), which correspond to the points of boundary $\mathbb{S}^{1}$ of the Poincaré disc, the infinite singular points are the solutions of the system

$$
\dot{u}=a u+c=0, \quad \dot{v}=0 .
$$

So, if $a \neq 0$ at infinity there is a unique singular point, namely $(u, 0)=$ $(-c / a, 0)$. When $a=0$ there are no infinite singular points in the local chart $U_{1}$ because $a^{2}+c^{2} \neq 0$.

At the singular point $(-c / a, 0)$ the Jacobian matrix has trace $a$ and determinant 0 . From section (1.5) in [18] we know that $(-c / a, 0)$ is a semi-hyperbolic singular point, and using Theorem 2.19 in [18] we obtain that it is a saddle-node such that when $c>0$ it has in $v>0$ the unstable parabolic sector, and in $v<0$ there are two hyperbolic sectors, recall that the infinity $v=0$ is invariant. When $c<0$ the sectors of the saddle-node interchange their localization with respect the line of the infinity.

Since the phase portrait in the local chart $V_{1}$ of the Poincaré sphere is the symmetric phase portrait with respect to the center of the sphere reversing the sign of all coefficients, so we have that the infinite singular point $(-c / a, 0)$ in $V_{1}$ when $c>0$ has in $v>0$ the two hyperbolic sectors, and in $v<0$ the parabolic sector. When $c<0$ the sectors of the saddle-node interchange their localization with respect the line of the infinity.

When $c=0$ the origin is the unique infinite singular point and it is a semi-hyperbolic singular point. Again, by Theorem 2.19 of [18] we obtain that $(0,0)$ is either a saddle if $a d<0$, or an unstable node if $a d>0$ or a saddle-node if $d=0$ and $e \neq 0$.

3.2. The origin of the local charts $U_{2}$ and $V_{2}$. Once we have studied the infinite singular points in the local charts $U_{1}$ and $V_{1}$, it only remains to study if the origin of the local charts $U_{2}$ and $V_{2}$ are infinite singular points.

Again using the results stated in [18], chapter 5, we obtain the expression of the Poincaré compactification $p(\mathcal{X})$ of system $(1)$ in the local chart $U_{2}$, i.e.

$$
\begin{aligned}
& \dot{u}=v-a u^{2}-b u v-c u^{3}-d u^{2} v-e u v^{2} \\
& \dot{v}=-a u v-b v^{2}-c u^{2} v-d u v^{2}-e v^{3}
\end{aligned}
$$



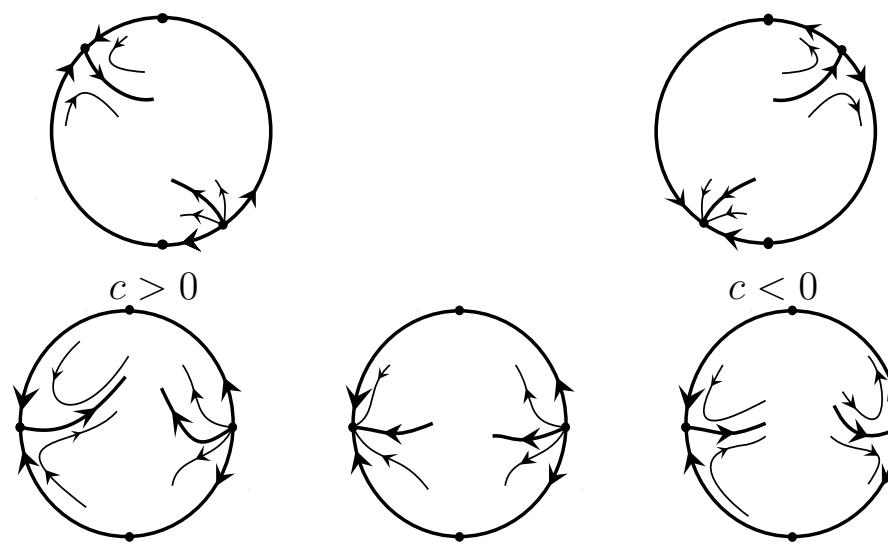

$$
c=d=0
$$

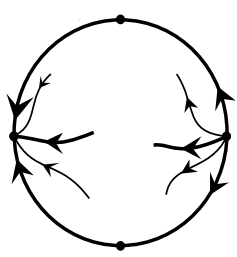

$c=0$ and

$a d>0$

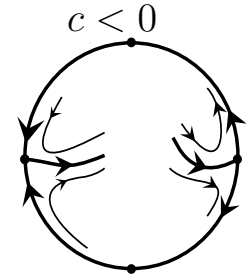

$c=0$ and

$a d<0$

FiguRE 1. Local phase portraits in the Poincaré disc at the chart $U_{1}$ and $V_{1}$.

Clearly the origin $(u, v)=(0,0)$ is an infinite singular point of this system.

Proposition 4. The local phase portrait at the origin of $U_{2}$ and $V_{2}$ is described in Figure 2 .

Proof. The Jacobian matrix of system (6) at the singular point $(0,0)$ has trace and determinant equal to zero, but it is not the zero matrix. So according to the definitions of section (1.5) in [18] the origin of system (6) is a nilpotent singular point, and we can study its local phase portrait using Theorem 3.5 of [18]. Doing that we get that if $a \neq 0$ then the local phase portrait at the origin is formed by two sectors one elliptic and one hyperbolic, of course separating these two sectors we can consider two parabolic sectors. When $a=0$ we have that $c \neq 0$, and then the origin is a stable node if $c>0$, and an unstable node if $c<0$.

In order to know the position of the invariant straight line of the infinity $v=0$ with respect to the elliptic and hyperbolic sectors when $a \neq 0$, we need to do the changes of variable known as blow up's, see for more details chapter 3 of [18]. Doing such changes we get that the elliptic sector is in $v>0$ and the hyperbolic sector is in $v<0$. Moreover there are no parabolic sectors in $v<0$.

In short we have completed the description of the local phase portraits at the infinite singular points. 


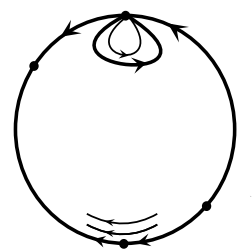

$a \neq 0$

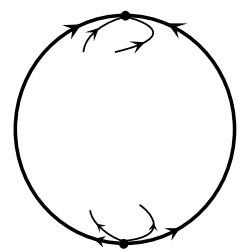

$a=0$ and

$c>0$

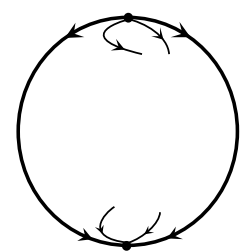

$a=0$ and

$c<0$

Figure 2. Local phase portraits in the Poincaré disc at chart $U_{2}$ and $V_{2}$.

\section{Finite Singular POINTS FOR EACH NORMAL FORM}

In this section we study the local behavior of the finite singular points for each normal form of system (1) presented in Table 1. To analyze the local phase portraits of every finite singular point we use the results of [18].

Case (i) Then system (1) is

$$
\dot{x}=y, \quad \dot{y}=x^{2}+x y+A x+B y+C .
$$

If $A^{2}-4 C<0$ this system has no finite singular points. Assume $A^{2}-4 C \geq 0$ and the finite singular points of system $(7)$ are $p_{ \pm}=$ $\left(\left(-A \pm \sqrt{A^{2}-4 C}\right) / 2,0\right)$, and the Jacobian matrix in each singular point has determinant $\Delta_{ \pm}=\mp \sqrt{A^{2}-4 C}$ and trace $T_{ \pm}=B+(-A \pm$ $\left.\sqrt{A^{2}-4 C}\right) / 2$.

When $A^{2}-4 C=0$ we get $\Delta=0$ and there is a unique singular point, that is, $p=(-A / 2,0)$ which is nilpotent if $T=B-A / 2=0$, or semi-hyperbolic if $T \neq 0$. Applying Theorem 2.19 of [18] in the semihyperbolic case we get that $p$ is a saddle-node. For the case nilpotent we apply Theorem 3.5 of [18] and conclude that $p$ is a cusp.

When $A^{2}-4 C>0$ there are two singular points, that is, $p_{ \pm}$. Since $\Delta_{+}<0$ we have that $p_{+}$is a saddle, and $p_{-}$is either a focus, or a node. If $T_{-}^{2}-4 \Delta_{-}<0$ then $p_{-}$is either a stable focus if $T_{-}<0$, or an unstable focus if $T_{-}>0$. If $T_{-}^{2}-4 \Delta_{-}>0$ then $p_{-}$is either a stable node if $T_{-}<0$, or an unstable node if $T_{-}>0$. See these local phase portraits in Figures 3, 4 and 5.

All these local phase portraits are achievable with the respective parameters shown in Table 2. 


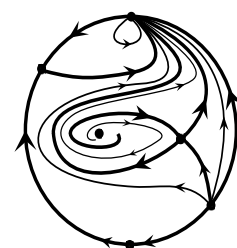

(a)

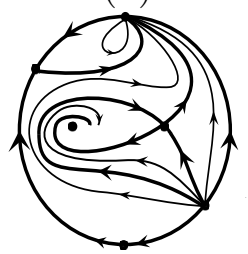

(d)

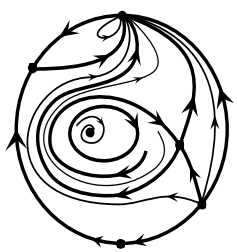

(b)

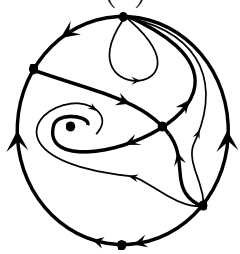

(e)

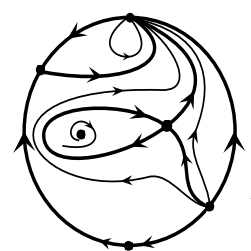

(c)

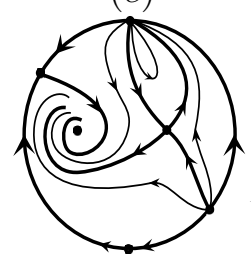

$(f)$

Figure 3. Local phase portraits in the Poincaré disc of Case (i) when $A^{2}-4 C>0$. In (a) $T_{-}>0$, in (b) $T_{-}=0$ and in (d), (e) and (f) $T_{-}<0$.

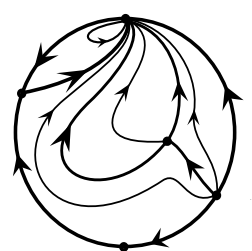

$$
B-A / 2>0
$$

(a)

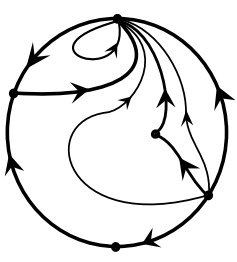

$B-A / 2=0$

(b)

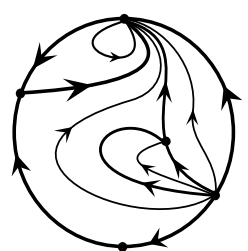

$B-A / 2<0$

(c)

Figure 4. Phase portraits in the Poincaré disc of Case (i) when $A^{2}-4 C=0$.

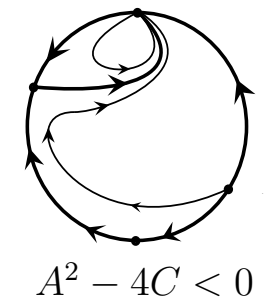

Figure 5. Phase portraits in the Poincaré disc of Case (i) when $A^{2}-4 C<0$.

As we can see in all the above local phase portraits there exists at most one limit cycle for the Liénard differential system that we are analyzing. This is a known result which can be found in [12]. More 


\begin{tabular}{|c|c|c|}
\hline \hline$A^{2}-4 C>0$ & $A=3, B=5, C=1$ & Figure 3 (a) \\
\cline { 2 - 3 } & $A=0, B=1, C=-3 / 2$ & Figure 3 (b) \\
\cline { 2 - 3 } & by continuity & Figure 3 (c) \\
\cline { 2 - 3 } & $A=3, B=2, C=1$ & Figure 3 (d) \\
\cline { 2 - 3 } & by continuity & Figure 3 (e) \\
\cline { 2 - 3 } & $\mathrm{A}=3, \mathrm{~B}=1, \mathrm{C}=1$ & Figure 3 (f) \\
\hline
\end{tabular}

\begin{tabular}{|l|l|c|c|}
\hline \hline$A^{2}-4 C=0$ & $B-A / 2>0$ & $A=-1, B=1 / 2, C=1 / 4$ & Figure 4 (a) \\
\cline { 2 - 4 } & $B-A / 2=0$ & $A=2, B=1, C=1$ & Figure 4 (b) \\
\cline { 2 - 4 } & $B-A / 2<0$ & $A=3, B=1, C=9 / 4$ & Figure 4 (c) \\
\hline \hline
\end{tabular}

$$
\begin{array}{|l|l|l|}
\hline \hline A^{2}-4 C<0 & A=2, B=1, C=2 & \text { Figure 5 } \\
\hline
\end{array}
$$

TABLE 2. Parameters of Case (i) realizing all the possible phase portraits in the Poincaré disc.

precisely, in [12], the author proved the following. By considering the Liénard equation

$$
x^{\prime \prime}-f(x) x^{\prime}+g(x)=0,
$$

or the equivalent system

$$
\begin{aligned}
& x^{\prime}=F(x)-y, \\
& y^{\prime}=g(x),
\end{aligned}
$$

where

$$
F(x)=\int_{0}^{x} f(\xi) d \xi
$$

he proved:

Theorem 5. Let $f$ and $g$ be continuously differentiable functions on the open interval $(a, b)$, where $a<0<b$, such that

(i) $g(x)>0$ (resp. $g(x)<0)$ according as $x>0$ (resp. $x<0)$,

(ii) $f(x)>0$ (resp. $g(x)<0$ ) according as $x>x_{0}$ (resp. $x<x_{0}$ ), where $x_{0}<0$,

(iii) the simultaneous equations

$$
\begin{aligned}
F\left(x_{1}\right) & =F\left(x_{2}\right) \\
f\left(x_{1}\right) / g\left(x_{1}\right) & =f\left(x_{2}\right) / g\left(x_{2}\right)
\end{aligned}
$$


have at most one solution $\left(x_{1}, x_{2}\right)=\left(\xi_{1}, \xi_{2}\right)$ with $a<\xi_{1}<x_{0}$ and $0<\xi_{2}<b$,

(iv) if $F\left(\xi_{0}\right)=0$ for some $\xi_{0}<x_{0}$, then $f(x) F(x) / g(x)$ is a decreasing function for $a<x<\xi_{0}$.

Then system (9) has at most one periodic orbit and, if it exists, it has a negative characteristic exponent, i.e. it is a stable limit cycle.

In this Case (i), when $\alpha^{2}=A^{2}-4 C, \alpha>0$ and $-A+2 B-\alpha=0$, the singular point $(-b, 0)$ is a weak focus. In fact, it is always an unstable focus, and never a center, since the first Lyapunov constant no zero is equal $\pi / 4 \beta^{5}>0$, with $\beta^{2}=\alpha$. As Theorem 5 ensures that the Liénard differential system that we are analyzing has at most one periodic orbit, with $B=\left(A+\sqrt{A^{2}-4 C}\right) / 2$, we have a Hopf bifurcation.

Case (ii) Then system (1) becomes

$$
\dot{x}=y, \quad \dot{y}=x^{2}+y+D x+E .
$$

The finite singular points of system $(11)$ are $p_{ \pm}=\left(\left(-D \pm \sqrt{D^{2}-4 E}\right) / 2,0\right)$, and the Jacobian matrix at $p_{ \pm}$is

$$
\left.J\right|_{p_{ \pm}}=\left(\begin{array}{cc}
0 & 1 \\
\pm \sqrt{D^{2}-4 E} & 1
\end{array}\right),
$$

which has trace $T=1$ and determinant $\Delta=\sqrt{D^{2}-4 E}$ for $p_{-}$, and $\Delta=-\sqrt{D^{2}-4 E}$ for $p_{+}$. First we observe the necessary condition $D^{2}-4 E>0$ for the existence of the equilibrium points $p_{ \pm}$. If $D^{2}-4 E=$ 0 we get a unique singular point which is semi-hyperbolic. Applying Theorem 2.19 of [18] at this semi-hyperbolic singular point we get that this singularity is a saddle-node.

If $D^{2}-4 E>0$ we obtain two singular points, i.e. $p_{ \pm}$. In this case $p_{+}$is a saddle because $T=1$ and $\Delta=-\sqrt{D^{2}-4 E}<0$, and the other equilibrium point $p_{-}$is an unstable node if $0<D^{2}-4 E \leq 1 / 16$, or an unstable focus if $D^{2}-4 E>1 / 16$. See these local phase portraits in Figure 6.

All these local phase portraits are achievable with the respective parameters shown in Table 3.

Case (iii) Then the correspondent normal form is

$$
\dot{x}=y, \quad \dot{y}=x y+y+G x+H .
$$




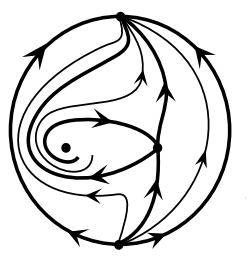

$D^{2}-4 E>0$

(a)

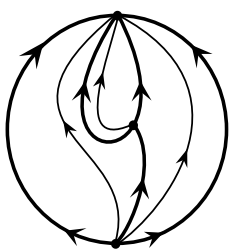

$D^{2}-4 E=0$

(b)

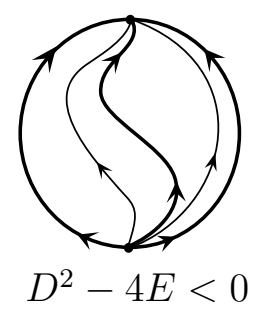

(c)

Figure 6. Phase portraits in the Poincaré disc of Case (ii).

\begin{tabular}{|c|c|l|}
\hline \hline$D^{2}-4 E>0$ & $D=3, E=2$ & Figure 6 (a) \\
\hline$D^{2}-4 E=0$ & $D=3, E=9 / 4$ & Figure 6 (b) \\
\hline$D^{2}-4 E<0$ & $D=3, E=3$ & Figure 6 (c) \\
\hline
\end{tabular}

TABLE 3. Parameters of Case (ii).

If $G=0$ there is no finite singular point for system (12). And, if $G \neq 0$, this system has a unique finite singular point $(-H / G, 0)$.

Consider $G \neq 0$, then the Jacobian matrix at this singular point $(-H / G, 0)$ is

$$
\left.J\right|_{(-H / G, 0)}=\left(\begin{array}{cc}
0 & 1 \\
G & 1-H / G
\end{array}\right) .
$$

Then $\left.J\right|_{(-H / G, 0)}$ has trace $T=1-H / G$ and determinant $\Delta=-G$. Hence, if $G>0$ then $\Delta<0$ and the singular point $(-H / G, 0)$ is a saddle. When $G<0$ we get $\Delta>0$ and in this case we have to analyse two possibilities. More precisely we can have

(i) $T^{2} \geq 4 \Delta \Leftrightarrow H^{2}-2 G H+G^{2}+4 G^{3} \geq 0$,

(ii) $4 \Delta>T^{2}>0 \Leftrightarrow H^{2}-2 G H+G^{2}+4 G^{3}<0$.

In case (i) the singular point is a node and in case (ii) the singular point is a focus. In both cases the singular point is stable if $H>G$ and unstable if $H<G$.

For all these local phase portraits see Figures 7 and 8 .

All these local phase portraits are achievable with the respective parameters shown in Table 4.

Case (iv) The correspondent normal form is

$$
\dot{x}=y, \quad \dot{y}=x y+x+I,
$$




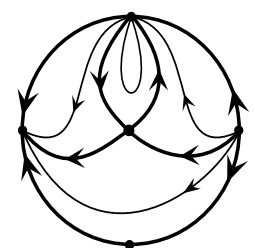

$G>0$

(a)

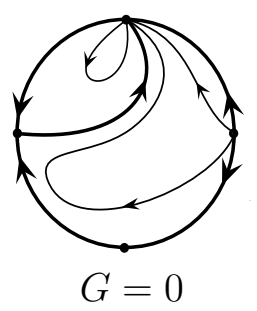

(b)

Figure 7. Phase portraits in the Poincaré disc of Case (iii) when $G \geq 0$.

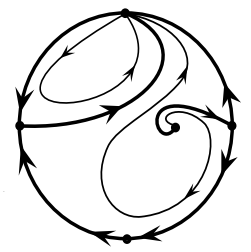

Figure 8. Phase portrait in the Poincaré disc of Case (iii) when $G<0$ and $G<H$. If $G>H$ then the phase portrait is the same but reversing the orientation of all its orbits.

\begin{tabular}{|c|c|c|}
\hline \hline$G>0$ & $G=1, H=1$ & Figure 7 (a) \\
\hline$G=0$ & $G=0, H=1$ & Figure 7 (b) \\
\hline$G<0$ & $G=-1, H=-2$ & Figure 8 \\
\hline \hline
\end{tabular}

TABle 4. Parameters of Case (iii).

which has a unique finite singular point $(-I, 0)$. The Jacobian matrix at this singular point is

$$
\left.J\right|_{(-I, 0)}=\left(\begin{array}{cc}
0 & 1 \\
1 & -I
\end{array}\right) .
$$

Since $\left.J\right|_{(-I, 0)}$ has trace $T=-I$ and determinant $\Delta=-1$ we conclude that the singular point $(-I, 0)$ is a saddle. This local phase portrait is topologically equivalent to that ones presented at Figure 7 (a).

Case (v) The correspondent normal form is

$$
\dot{x}=y, \quad \dot{y}=x y-x+I,
$$


which has a unique finite singular point $(I, 0)$. The Jacobian matrix at this singular point is

$$
\left.J\right|_{(I, 0)}=\left(\begin{array}{cc}
0 & 1 \\
-1 & I
\end{array}\right) .
$$

which has trace $T=I$ and determinant $\Delta=1$. If $I=0$ then the singular point $(0,0)$ is a center non-hyperbolic because in this case the system is reversible with involution given by $(x, y, t) \mapsto(-x, y,-t)$ (see Figure 9$)$. If $I \neq 0$ there exist two possibilities

(i) $T^{2} \geq 4 \Delta>0 \Leftrightarrow I^{2} \geq 4>0 \Leftrightarrow I \leq-2$ or $I \geq 2$,

(ii) $4 \Delta>T^{2}>0 \Leftrightarrow 4>I^{2} \Leftrightarrow-2<\bar{I}<2$.

In case (i) the singular point $(I, 0)$ is a stable node if $I \leq-2$, or an unstable node if $I \geq 2$. In case (ii) the singular point $(I, 0)$ is a stable focus if $-2<I<0$, or an unstable focus if $0<I<2$. These local phase portraits are topologically equivalent to that ones presented at Figure 8.

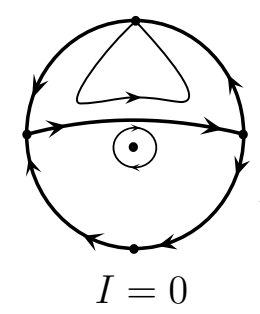

Figure 9. Phase portraits in the Poincaré disc of Case (v) when $I=0$.

Case (vi) The correspondent normal form is

$$
\dot{x}=y, \quad \dot{y}=x y+1,
$$

which has no finite singular point. This local phase portrait is topologically equivalent to the one of Figure 5.

\section{ACKNOWLEDGEMENTS}

The first and the third authors are partially supported by PROCADCAPES grant 88881.068462/2014-01 and by FAPESP grant 2013/245410 . The second author is partially supported by the Ministerio de Ciencia, Innovación y Universidades, Agencia Estatal de Investigación grants MTM2016-77278-P (FEDER) and MDM-2014-0445, the Agència de Gestió d'Ajuts Universitaris i de Recerca grant 2017SGR1617, and the H2020 European Research Council grant MSCA-RISE-2017-777911. 


\section{REFERENCES}

[1] V. I. ARnold And Y. S. Ilyashenko, Dynamical Systems I, Ordinary Differential Equations". Encyclopaedia of Mathematical Sciences, Vols 1-2, SpringerVerlag, Heidelberg, 1988.

[2] J.C. ARTÉS AND J. Llibre, Hamiltonian quadratic systems, J. Differential Equations 107 (1994), 80-95.

[3] J.C. ARTÉS AND J. LliBRE, Phase portraits for quadratic systems having a focus and one antisaddle, Rocky Mountain J. Math. 24 (1994), 875-889.

[4] J.C. ARTÉs AND J. LliBRe, Quadratic vector fields with a weak focus of third order, Publicacions Matemàtiques 41 (1997), 7-39.

[5] J.C. Artés, J. Llibre And D. SChlomiuk, The geometry of the quadratic differential systems with a weak focus of second order, Int. J. of Bif. and Chaos 16 (2006), 3127-3194.

[6] A.N. Berlinskit, Qualitative study of the differential equation $x^{\prime}=x+b_{0} x^{2}+$ $b_{1} x y+b_{2} y^{2}, y^{\prime}=y+a_{0} x^{2}+a_{1} x y+a_{2} y^{2}$, Differ. Equ. 2 (1966), 174-178.

[7] J. Chavarriga, I.A. García, J. Llibre And H. Zoladek, Invariant algebraic curves for the cubic Liénard system with linear damping, Bull. Sci. Math. 130 (2006), no. 5, 428-441.

[8] L.A. CherKAs, Liénard systems for quadratic systems with invariant algebraic curves, Differ. Equ. 47 (2011), 1435-1441

[9] G. Chèze and T. Cluzeau, On the nonexistence of Liouvillian first integrals for generalized Liénard polynomial differential systems, J. Nonlinear Math. Phys. 20 (2013), no. 4, 475-479.

[10] C. ChICone, Quadratic gradients on the plane are generically Morse-Smale, J. Differential Equations 33 (1979), 159-166.

[11] B. Coll, A. Gasull And J. Llibre, Some theorems on the existence, uniqueness and non-existence of limit cycles for quadratic systems, J. Differential Equations 67 (1987), 372-399.

[12] W. A. Coppel, Some quadratic systems with at most one limit cycles, Dynamics Reported Vol. 2, Wiley, (1998), 61?-68.

[13] T. DATE, Classification and analysis of two-dimensional homogeneous quadratic differential equations systems, J. of Differential Equations 32 (1979), 311-334.

[14] P. De Maesschalck And F. Dumortier, Classical Liénard equations of degree $n=6$ can have $[(n-1) / 2]+2$ limit cycles, J. Differential Equations 250 (2011), 2162-2176

[15] R.J. Dickson And L.M. Perko, Bounded quadratic systems in the plane, J. Differential Equations 6 (1970), 251-273.

[16] F. Dumortien, Sharp upperbounds for the number of large amplitude limit cycles in polynomial Lienard systems, Discrete Contin. Dyn. Syst. 32 (2012), 1465-1479.

[17] F. Dumortier, C. Herssens And L. Perko, Local bifurcations and a survey of bounded quadratic systems, J. Differential Equations 165 (2000), 430-467.

[18] F. Dumortier, J. Llibre And J.C. Artés, Qualitative theory of planar differential systems, Springer-Verlag, Berlin, Heidelberg, 2006.

[19] F. Dumortier, D. Panazzolo and R. Roussarie, More limit cycles than expected in Liénard equations, Proc. Amer. Math. Soc. 135 (2007), 1895-1904. 
[20] A. Gasull, S. Li-Ren And J. Llibre, Chordal quadratic systems, Rocky Mountain J. of Math. 16 (1986), 751-782.

[21] A. Gasull AND J. LliBRe, On the nonsingular quadratic differential equations in the plane, Proc. Amer. Math. Soc. 104 (1988), 793-794.

[22] D.D. Hua, L. Cairó, M.R. Feix, K.S. Govinder, P.G.L. Leach, Connection between the existence of first integrals and the Painlevé property in two-dimensional Lotka-Volterra and quadratic systems, Proc. Roy. Soc. London Ser. A 452 (1996), 859-880.

[23] C. Li AND J. LliBre, Uniqueness of limit cycles for Liénard differential equations of degree four, J. Differential Equations 252 (2012), 3142-3162.

[24] A. Lins, W. DE Melo And C.C. Pugh, On Liénard's equation, Geometry and topology (Proc. III Latin Amer. School of Math., Inst. Mat. Pura Aplicada CNPq, Rio de Janeiro, 1976), pp. 335-357. Lecture Notes in Math., Vol. 597, Springer, Berlin, 1977.

[25] C. Liu, G. Chen And J. YAng, On the hyperelliptic limit cycles of Liénard systems, Nonlinearity 25 (2012), 1601-1611.

[26] J. LliBRE AND D. SChlOMiUK, The geometry of differential quadratic systems with a weak focus of third order, Canadian J. of Math. 56 (2004), 310-343.

[27] J. LlibRe AND C. VALLS, Liouvillian first integrals for generalized Liénard polynomial differential systems, Adv. Nonlinear Stud. 13 (2013), 825-835.

[28] J. Llibre AND X. Zhang, On the algebraic limit cycles of Liénard systems, Nonlinearity 21 (2008), 2011-2022.

[29] V.A. Lunkevich And K. S. SibirskiI, Integrals of a general quadratic differential system in cases of a center, Differ. Equ. 18 (1982), 563-568.

[30] L. Markus, Global structure of ordinary differential equations in the plane: Trans. Amer. Math Soc. 76 (1954), 127-148.

[31] L. MARKus, Quadratic differential equations and non-associative algebras, Annals of Mathematics Studies 45, Princeton University Press, 1960, pp 185-213.

[32] D. A. Neumann, Classification of continuous flows on 2-manifolds, Proc. Amer. Math. Soc. 48 (1975), 73-81.

[33] T.A. Newton, Two dimensional homogeneous quadratic differential systems, SIAM Review 20 (1978), 120-138.

[34] K. ODANI, The limit cycle of the van der Pol equation is not algebraic, J. Differential Equations 115 (1995), 146-152.

[35] M.M. Peiхото, Dynamical Systems. Proccedings of a Symposium held at the University of Bahia, 389-420, Acad. Press, New York, 1973.

[36] S. Rebollo-Perdomo, Medium amplitude limit cycles of some classes of generalized Liénard systems, Internat. J. Bifur. Chaos Appl. Sci. Engrg. 25 (2015), no. 10, 1550128, 8 pp.

[37] J. REYN, Phase portraits of planar quadratic systems, Mathematics and Its Applications 583, Springer, New York, 2007.

[38] V. Romanovski, M. Han and N. Li, Cyclicity of some Liénard Systems, Commun. Pure Appl. Anal. 14 (2015), 2127-2150.

[39] I.G. Roset, Nonlocal bifurcation of limit cycles and quadratic differential equations in the plane (in Russian), Samarkand University, Dissertation kand. phys. mat., 1991. 
[40] J. Shen And M. HAn, Bifurcations of canard limit cycles in several singularly perturbed generalized polynomial Liénard systems, Discrete Contin. Dyn. Syst. 33 (2013), 3085-3108.

[41] N.I. VulPe, Affine-invariant conditions for the topological discrimination of quadratic systems with a center, Differ. Equ. 19 (1983), 273-280.

[42] L. YANG AND X. ZENG, The convexity of closed orbits of Liénard systems, Bull. Sci. Math. 137 (2013), no. 2, 215-219.

[43] L. Yang And X. Zeng, The period function of Liénard systems, Proc. Roy. Soc. Edinburgh Sect. A 143 (2013), 205-221.

[44] Ye Yanqian, Theory of limit cycles, Translations of Math. Monographs, Amer. Math. Soc., Vol 66, 1986.

[45] H. ZoladeK, Algebraic invariant curves for the Liénard equation, Trans. Amer. Math. Soc. 350 (1998), 1681-1701.

1 Departamento de Matemática, Ibilce-UNESP, 15054-000 São José do Rio Preto, Brasil

E-mail address: mra.gouveia@unesp.br, luci.roberto@unesp.br

2 Departament de Matemàtiques, Universitat Autònoma de Barcelona, 08193 Bellaterra, Barcelona, Catalonia, Spain

E-mail address: jllibre@mat.uab.cat 